\title{
BARRERAS PARA IMPLEMENTAR EL DESPISTAJE, INTERVENCIONES BREVES Y REFERENCIA AL TRATAMIENTO POR PROBLEMAS DE CONSUMO DE ALCOHOL Y OTRAS DROGAS EN HOSPITALES QUE ATIENDEN PERSONAS QUE VIVEN CON EL VIH/SIDA EN EL PERÚ
}

\author{
Kim A Hoffman 1,2,a, Jessica Beltrán ${ }^{3, b}$, Javier Ponce 1,5,b , Lisset García-Fernandez ${ }^{4, b}$, María Calderón ${ }^{6, b}$, \\ John Muench ${ }^{2, b}$, Carlos Benites ${ }^{3, b}$, Leslie Soto ${ }^{6, b}$, Dennis McCarty ${ }^{2, c}$, Fabián Fiestas $^{7, b}$
}

\begin{abstract}
RESUMEN
Objetivos. Evaluar la factibilidad de implementar la detección, intervención breve y referencia a tratamiento (SBIRT, por sus siglas en inglés) en los servicios de atención para personas viviendo con el virus de inmunodeficiencia humana/ síndrome de inmunodeficiencia adquirido (PVVS). Materiales y métodos. Se realizó un estudio de tipo cualitativo, luego de capacitar profesionales de salud (incluyendo, enfermeras y médicos) que proveen atención de salud a PVVS en la aplicación del SBIRT, se realizaron grupos focales para explorar las barreras percibidas para su implementación y se realizaron entrevistas para evaluar las barreras y facilitadores en dos hospitales de tercer nivel de Lima, Perú. Resultados. La codificación temática de los grupos focales y las entrevistas revelaron tres dimensiones principales de barreras: 1) el desconocimiento acerca del consumo de sustancias en las PVVS; 2) limitaciones de espacio y tiempo que dificultan las intervenciones breves durante las visitas de rutina, y 3 ) insuficientes servicios con capacidades para el tratamiento el uso de sustancias apropiadas donde puedan ser referidos los pacientes con estos problemas. Conclusiones. Múltiples barreras, incluyendo el desconocimiento por los problemas de consumo de sustancias, limitaciones de espacio y tiempo de los profesionales, y carencia de servicios especializados a donde referir los pacientes para un cuidado especializado, haría difícil implementar SBIRT en un contexto como el del sistema de salud peruano.
\end{abstract}

Palabras clave: Abuso de alcohol y otras drogas; intervención breve; VIH/SIDA (fuente: DeCS BIREME).

\section{BARRIERS TO IMPLEMENTING SCREENING, BRIEF INTERVENTION AND REFERRAL TO TREATMENT FOR SUBSTANCE USE IN HIV/AIDS HEALTH SERVICES IN PERU}

\begin{abstract}
Objectives. Screening and treatment for substance use among people living with HIVIAIDS (PLWHA) is highly recommended. Nevertheless, in Peru healthcare for PLWHA does not include a standardized or systematic assessment to identify substance use. The aim of this study was to assess the feasibility of implementing screening, brief intervention and referral to treatment (SBIRT) in healthcare settings attending people living with PLWHA. Materials and methods. After providing training in SBIRT for PLWHA's healthcare personnel (including nurses and physicians) focus groups were conducted to explore knowledge, beliefs and perceived barriers to implementation and interviews were conducted to assess the barriers and facilitators of two tertiary hospitals in Lima, Peru. Results. focus groups and interviews' thematic coding revealed three dimensions: 1) the unknown extent of substance use within PLWHA, 2) space and time limitations hinder completion of brief interventions during routine visits, and 3 ) insufficient access to substance use treatment appropriate for HIV patients. Conclusions. Multiple barriers, including lack of awareness of substance use problems, limited space and time of providers, and lack of specialized services to refer patients for treatment make it difficult to implement SBIRT in the Peruvian healthcare system.
\end{abstract}

Key words: Alcohol and drug abuse; brief intervention; HIVIAIDS (source: MeSH NLM).

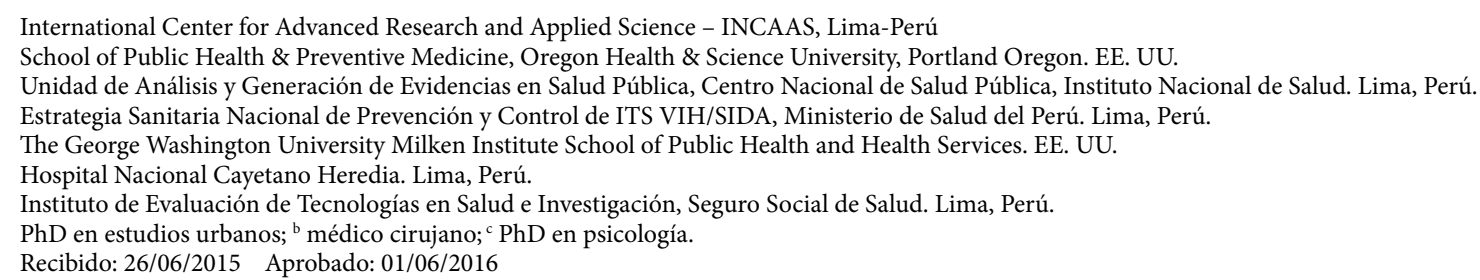

Citar como: Hoffman KA, Beltrán J, Ponce J, García-Fernandez L, Calderón M, Muench J, et al. Barreras para implementar el despistaje, intervenciones breves y referencia al tratamiento por problemas de consumo de alcohol y otras drogas en hospitales que atienden personas que viven con el VIH/SIDA en el Perú. Rev Peru Med Exp Salud Publica. 2016;33(3):432-7. doi: 10.17843/rpmesp.2016.333.2293 


\section{INTRODUCCIÓN}

Los trastornos de uso de alcohol y drogas tienen un efecto negativo en el curso natural de la enfermedad de las personas viviendo con el virus de inmunodeficiencia humana/síndrome de inmunodeficiencia adquirido (PVVS). El trastorno por uso de sustancias interfiere con el alcance de los resultados clínicos del tratamiento antirretroviral (TARGA), que incluye la falla en la recuperación del conteo de CD4 y en la supresión viral ${ }^{(1,2)}$. Asimismo, disminuye la adherencia al TARGA y reduce el uso de servicios de salud (1,3-5). Además, el trastorno por uso de sustancias en PVVS se ha asociado con otras comorbilidades, como el aumento en la incidencia de polineuropatía distal simétrica ${ }^{(6)}$, alteración de la función neurocognitva ${ }^{(7,8)}$, depresión ${ }^{(9)}$, lipodistrofia ${ }^{(10)} \mathrm{y}$, en casos de coinfección con el virus de la hepatitis $\mathrm{C}(\mathrm{VHC})$, una progresión acelerada hacia la insuficiencia hepática terminal (11). Por otro lado, los trastornos por uso de sustancias en PVVS se asocian a conductas sexuales de riesgo (i.e. relaciones sexuales sin protección y múltiples parejas sexuales) ${ }^{(12)}$, y también podrían llevar a una demora en el diagnóstico ${ }^{(13)}$. De esta forma, todos los factores antes mencionados aceleran la progresión de la enfermedad por $\mathrm{VIH}$, aumentan la mortalidad y la carga de enfermedad relacionada al $\mathrm{VIH}$, e incrementan la transmisión de la infección por $\mathrm{VIH}^{(1,14)}$.

En base a las recomendaciones internacionales y nacionales, la atención a PVVS debería incluir un despistaje de uso de sustancias ${ }^{(15)}$. En el Perú, el despistaje de uso de sustancias no se realiza de una manera sistemática ni estandarizada, tampoco se utilizan frecuentemente instrumentos validados. Ante esta brecha en la atención de PVVS, se propone una intervención basada en la detección, intervención breve y referencia al tratamiento (SBIRT, por sus siglas en inglés). Esta es una práctica clínica basada en la evidencia usada para identificar, reducir y prevenir trastornos por uso de sustancias, así como en aquellos que están en riesgo de desarrollarlos. EI SBIRT inicia con el despistaje, que consiste en que el profesional de la salud evalúa al paciente para conductas de uso riesgoso de sustancias empleando herramientas de despistaje estandarizadas. A los pacientes que dieron positivo en el despistaje, se les determina si necesitan de una intervención breve, tratamiento breve, o referencia a tratamiento especializado, según la severidad del puntaje obtenido en el despistaje.

La intervención breve utiliza los principios de la entrevista motivacional. La entrevista motivacional es un "estilo de asistencia directa centrada en el cliente, para provocar un cambio en el comportamiento, ayudando a los clientes a explorar y resolver ambivalencias" (16). La ambivalencia es un estado natural de incertidumbre que se produce debido a sentimientos contradictorios sobre el proceso y los resultados del cambio, y es un fenómeno común en el contexto del comportamiento adictivo. Los pacientes se sienten indecisos entre las consecuencias agradables y desagradables del consumo de drogas. El mensaje básico de la entrevista motivacional es que la intervención y la prevención efectiva es posible en una fase muy temprana, cuando los afectados aún no han desarrollado un interés por la asistencia o el cambio. La entrevista motivacional sostiene que el cambio de comportamiento es causado por la verbalización de los pacientes de sus propios argumentos para el cambio ("conversación del cambio") (17).

La entrevista motivacional ha probado ser eficaz en lograr un cambio de conducta en pacientes que tienen sentimientos en conflicto sobre el proceso y los resultados de dicho cambio, esto se conoce como ambivalencia ${ }^{(18,19,20)}$, y en poblaciones especiales, como las PVVS, ha demostrado mayor eficacia en la reducción de alcohol que en otras drogas ${ }^{(21)}$. A pesar de la eficacia probada del SBIRT, su implementación en el ámbito de la atención en salud ha encontrado muchas barreras, que incluyen la falta de recursos o incentivos económicos para el personal de salud por la realización de la intervención, el entrenamiento inadecuado de los proveedores, la atención inapropiada para los pacientes, la sobrecarga de trabajo, el tiempo insuficiente para la intervención ${ }^{(22)}$, y la falta de aceptabilidad de los pacientes y de los proveedores ${ }^{23,24)}$. Con el objetivo de evaluar las barreras subyacentes y los potenciales facilitadores de la implementación del SBIRT en los servicios de enfermedades infecciosas donde se atienden PVVS de dos hospitales en Lima, Perú, se realizó este estudio de factibilidad donde se usó un análisis cualitativo.

\section{MATERIALES Y MÉTODOS}

\section{DISEÑO DEL ESTUDIO}

En este estudio de factibilidad se utilizó una metodología cualitativa de tipo grupo focal y entrevista a profundidad para explorar las oportunidades y retos que se experimentaron al momento de implementar el SBIRT en el contexto actual del cuidado de PVVS en dos hospitales públicos del Perú ${ }^{(25,26)}$.

\section{EL ÁMBITO DE LA SALUD}

Este estudio se basa en las actividades hospitalarias establecidas para el cuidado de PVVS y que son delineadas normativamente por la Estrategia Sanitaria Nacional de Prevención y Control de Infecciones de Transmisión Sexual y VIH/SIDA (ESN PC ITS VIH/SIDA), enmarcadas dentro del modelo de atención integral de la salud. El estudio se realizó en dos hospitales de tercer nivel en Lima. Ambos hospitales cuentan con 
un departamento de enfermedades infecciosas, en los cuales se brinda la atención integral a las PVVS, entre los que se encuentran: la evaluación integral de PVVS, la administración del tratamiento antirretroviral, y la prevención y el tratamiento de las comorbilidades y otras infecciones de transmisión sexual. Ambos departamentos de enfermedades infecciosas cuentan con equipos multidisciplinarios conformados por los médicos especialistas en infectología, enfermeras, un psicólogo, una trabajadora social, una obstetra, entre otros profesionales de la salud. De acuerdo con información del monitoreo de la Estrategia Sanitaria Nacional de Prevención y Control de las ITS, VIH/ SIDA, en el año 2013, en uno de los hospitales se atendieron 2461 PVVS y se les administró la terapia antirretroviral a 1875 pacientes. Asimismo, en el otro hospital, en el mismo año, se atendieron 3273 PVVS y se les administró la terapia antirretroviral a 2676 pacientes.

\section{PROCEDIMIENTOS}

Los investigadores del Instituto Nacional de Salud, de la Oregon Health \& Science University (OHSU) y de dos hospitales referenciales colaboraron para la implementación del SBIRT por los proveedores de la salud que rutinariamente atienden a PVVS. Se tradujo y adaptó al contexto peruano el plan de estudios que la OHSU utiliza para capacitar en SBIRT a sus residentes de especialidades médicas. Con dicho plan, dos de los investigadores (JM y $\mathrm{KH}$ ) capacitaron en SBIRT a profesionales de los servicios de infectología de los dos hospitales participantes, para que incorporen el SBIRT a su práctica clínica habitual.

Para identificar las barreras de implementación del SBIRT en la práctica clínica se utilizó la metodología cualitativa, tanto del tipo de grupos focales, como de entrevistas a profundidad. Así, se realizaron dos grupos focales en los hospitales de estudio (hospital $1 \mathrm{~N}=31$; hospital $2 \mathrm{~N}=20$ ) antes de la capacitación del SBIRT. Los grupos focales, realizados antes de la capacitación, exploraron los conocimientos, las creencias, y las barreras percibidas para la implementación. Tres meses después de la implementación, se realizaron seis entrevistas semiestructuradas en los hospitales del estudio (hospital 1, N=3; hospital 2, N=3). Las preguntas posteriores a la implementación se centraron en la evaluación de las barreras y los facilitadores para la implementación del SBIRT para PVVS y en la factibilidad que la implementación se convierta en una práctica sostenible en sus respectivos establecimientos de salud. Las entrevistas fueron grabadas, transcritas y analizadas mediante análisis de contenido a través de Atlas.ti.

La codificación abierta se realizó por tres lectores que revisaron las transcripciones y discutieron los temas. Utilizando un enfoque de análisis del contenido, los lectores refinaron sus nociones acerca de los temas y las formas posibles de codificación de las respuestas. Los códigos temáticos se desarrollaron inductivamente según se revisaron y discutieron las transcripciones.

\section{RESULTADOS}

Los temas principales de los entrevistados revelaron tres dimensiones principales de barreras: las barreras estructurales y de los conocimientos; las barreras para completar los instrumentos y las intervenciones breves; y las barreras relacionadas con el sistema de referencia al tratamiento especializado.

\section{LAS BARRERAS ESTRUCTURALES Y DEL CONOCIMIENTO}

La barrera principal identificada en el dominio estructural y del conocimiento fue que se desconoce la magnitud real de los trastornos de uso de sustancias entre las PVVS en los hospitales de Lima. Por ejemplo, al preguntarle a un proveedor de salud sobre el porcentaje o el número de pacientes que actualmente consume alcohol $u$ otras drogas, este refirió: No, no podría decirle. La verdad es que no podría calcular el porcentaje, pero... un buen grupo de pacientes me ha referido haber probado algunos tipos de drogas.... Al no existir la información de la epidemiología del uso de sustancias en PVVS, los proveedores de salud solo pueden hacer estimaciones gruesas de acuerdo a su experiencia con los pacientes. Por ejemplo, durante los grupos focales de la etapa de la preimplementación, algunos médicos refirieron que el abuso de sustancias se encontraba en aproximadamente $10 \%$ de los pacientes, mientras que otros asumieron que los pacientes dejaban de consumir después de iniciar la terapia antirretroviral Al preguntárseles sobre la precepción general del nivel de consumo de drogas en las PVVS, respondieron: Los pacientes, normalmente, solo responden sí o no cuando se les pregunta por el consumo del alcohol o por el uso del tabaco. Pero siempre refieren haber dejado de consumir luego de iniciar el tratamiento... sobre el uso de drogas, se les pregunta, pero de nuestros pacientes, los consumidores activos no son muchos. Pero muchos de ellos tienen una historia pasada de consumo de drogas. Otro participante refirió: Los problemas relacionados al consumo de sustancias no representan un problema en este tipo de pacientes...eso es lo que yo he podido identificar, de la mayoría de los pacientes que veo uno o dos usarán drogas, Yo no identifico esto como un problema; no es muy común al menos en la población que yo atiendo.

A pesar de que todos los proveedores de atención de la salud refieren que "preguntan" sobre el consumo de sustancias, no existe una forma estandarizada de abordar este tema, lo que lleva a que no se pueda sistematizar la información como para conocer la 
magnitud del problema entre las PVVS atendidas en los servicios de infectología. Por ejemplo, durante los grupos focales de la etapa de preimplementación, los proveedores de la salud refirieron: $A$ todos se les pregunta. En la historia clínica se marca como sí o no. Pero es rápido, está escrito 'alcohol'y 'drogas', luego tú marcas sí o no. Le preguntas si usa o no usa drogas y marcas tu respuesta al costado. Si la respuesta del paciente es positiva (si dice que sí), entonces le preguntas por la frecuencia de uso, etc. Asimismo, en una de las entrevistas posimplementación, además, se mencionó que dependía de cada proveedor de la salud el decidir si preguntaba o no sobre el consumo de sustancias y que las historias clínicas no están diseñadas para registrar los resultados de SBIRT, además que un instrumento validado para evaluar el nivel del riesgo para la dependencia no es parte de la historia clínica o formatos que se llenan cuando un PVVS inicia su manejo en el servicio de salud: Es que nosotros tenemos un formato...este es el formato [entrevistado sostiene en la mano el formato donde no se establece una forma estandarizada de preguntar por el consumo de sustancias]...,pero no tenemos un formato estándar que todos los médicos manejemos para los pacientes nuevos, no está prehecho, o sea, no hay una ficha para todos. Entonces si a mí me parece le pregunto [por el consumo de sustancias], si no, no. Lo que tú preguntas yo no pregunto. O sea, ¿me entiendes?, no hay algo que está escrito y que todo paciente nuevo [debe llenar]... [...] esta es la ficha de inicio de TARGA y aquí en ningún momento dice "consumo de drogas"...

Otra barrera importante es que los proveedores de atención de la salud que atienden PVV no han recibido la capacitación en la detección del trastorno de uso de sustancias. Ellos no conocen ni manejan los instrumentos validados para hacer el despistaje. Además, se resalta la necesidad de reforzar las definiciones conceptuales del uso, el abuso y la dependencia de drogas y del alcohol.: Si, preguntamos si los pacientes toman alcohol, pero en mi opinión, creo que no tenemos definido el (nivel) de consumo del alcohol. Por ejemplo, qué es "bebedor social", o sea, ¿toma todos los días?, ¿una vez al mes? Tal vez eso es lo hay que definir... ¿qué es consumo del alcohol?

\section{LAS BARRERAS PARA COMPLETAR LAS INTERVENCIONES BREVES}

En cuantoa las barreras para completar las intervenciones breves, encontramos las barreras logísticas y las ambientales, como la falta de infraestructura física que no permite tener un lugar confidencial para realizar el despistaje o intervenir en la consulta regular de la atención a los pacientes en TARGA. Otra barrera es que, en el caso de los pacientes en el tratamiento ambulatorio, especialmente los de larga data, ni los pacientes ni los médicos tienen tiempo para administrar los instrumentos, ni las entrevistas motivacionales durante los chequeos. Las consultas médicas de las PVVS son a menudo muy cortas, ya que muchos se encuentran en una condición estable y solo vienen para el control de laboratorio y para recibir sus medicamentos. Asimismo, a veces son los parientes de los pacientes los que vienen a recoger el medicamento en lugar de ellos, lo que imposibilita la aplicación del SBIRT. Por ejemplo: Los médicos no tienen ni la voluntad ni el tiempo de hacerlo, ¿no es cierto? He intentado hacerlo y a mí me ha demandado tiempo porque son varias respuestas [...] Entonces... te quita tiempo ...el problema es que acá uno avanza, avanza, avanza, porque hay gente que hay que hacerle TARGA, hay que entregarle órdenes, entonces como que (el SBIRT) no se adapta a nuestro sistema, se hace más complicado. Quizás en la atención primaria, quizás haya más tiempo o en otro consultorio, o acortarlo no sé. Es que son varias etapas (acerca del SBIRT).

Además, algunos de los médicos entrevistados revelaron que el despistaje, la intervención breve y la referencia al tratamiento en las PVVS, no es realmente su "función". Cuando se les preguntó si tal vez las enfermeras podrían tener mayor disponibilidad de tiempo para aplicar el SBIRT en vez de los médicos, los entrevistados respondieron que el tiempo constituía una barrera para ellas también: El problema es que acá estamos llenos de pacientes que ya están estables, y el paciente también quiere irse porque tiene que trabajar, y las enfermeras igual están saturadas ... entonces, el paciente, incluso algunas veces, tiene que esperar dos horas para que le llame la enfermera. Quizás la que dispondría más tiempo es la psicóloga porque ella sí entra en conversación con el paciente.

Por otro lado, refirieron que cuando el paciente es nuevo, generalmente la primera visita es más prolongada, lo que representaría una oportunidad importante para superar la barrera relacionada al tiempo para la aplicación del SBIRT: Más o menos he calculado yo que me lleva de 10 a 15 minutos (llenar el formato), porque hay que explicarle el formato al paciente... Dependiendo de la situación, si estamos hablando de una atención regular de un paciente estable que viene a recoger medicamentos y ver cómo va, entonces probablemente sí sea el 50\% de su tiempo. Ya que estamos hablando de una evaluación de 10 a 15, el ASSIST puede tomar el $50 \%$ de su tiempo. Pero si estamos hablando de un paciente nuevo, entonces tomaría el $10 \%$ del tiempo de la consulta a lo mucho... eso facilita mucho.

\section{BARRERAS PARA LA REFERENCIA A TRATAMIENTO ESPECIALIZADO}

En cuanto a las barreras asociadas a las referencias al tratamiento contra las drogas y el alcohol, no hay una red 
establecida para remitir a los pacientes. Los pocos lugares disponibles, a menudo presentan largos tiempos de espera para los pacientes que lo necesitan. Otra preocupación importante está en relación a algunas prácticas clínicas controversiales en uno de los sitios de referencia (por ejemplo, la práctica de técnicas de aislamiento de severo y prolongados). Uno de los proveedores refirió: La verdad es que yo pienso que no hay muchas alternativas en cuanto al tratamiento de un paciente con problemas de adicciones. El consultorio de psiquiatría del hospital no maneja pacientes con adicciones y todos van al (nombre de hospital de referencia)... otro hospital es el (nombre del otro hospital de referencia) que tiene hospitalización y maneja el tema de las adicciones, está repleto, colapsado. Entonces, realmente no hay muchas opciones para este tipo de pacientes.

\section{DISCUSIÓN}

Implementar el SBIRT en un contexto como el descrito representa un desafío debido a una variedad de factores, que incluyen la falta de conocimiento de la magnitud y de las características del consumo de drogas y alcohol en PVVS, la dificultad para que los profesionales de la salud puedan realizar los despistajes y las intervenciones breves, y la falta de un buen sistema de referencia y contrarreferencia para el tratamiento de dependencia a sustancias (i.e., adicción). Los resultados nos indican que no es posible implementar esta iniciativa en el contexto actual. Estos resultados son el reflejo de un sistema de salud que no tiene las condiciones o las estrategias para abordar el uso de sustancias entre las PVVS. Este estudio es el primer paso en identificar las barreras para una implementación exitosa del SBIRT en Perú.

La principal limitación de este estudio es que incluye solo dos centros. Se podría asumir que la población de pacientes que se atienden en los servicios de infectología de los hospitales del estudio no es representativa de todos los PVVS de todo el país. Sin embargo, estos establecimientos de salud son dos de los principales centros que brindan TARGA en Lima y cubren casi un tercio de las PVVS en esta provincia. También es importante recordar que el $70 \%$ de las PVVS viven en Lima. Así, el hecho de que el presente estudio se haya realizado en estos dos centros hospitalarios capitalinos, nos hace tener confianza que se está capturando una imagen general de qué tan factible es el implementar una metodología como el SBIRT en el sistema de salud peruano para pacientes con alto riesgo de consumo de sustancias, como son las PVVS.

En estudios previos, el principal problema con respecto a la aceptabilidad del paciente para la intervención fue el contexto de la aplicación ${ }^{(27)}$. En nuestro estudio también se encontró que el contexto de la aplicación no fue el más apropiado, debido a que la confidencialidad no estuvo garantizada por la falta de un espacio físico para la atención de los pacientes y, por ende, aplicar el SBIRT. Además, el hecho de realizar esta intervención durante una visita de rutina a un paciente estable, puede parecerle inusual o pérdida de tiempo. Una solución a este problema sería lo que se ha observado en estudios anteriores, aplicar el SBIRT en la consulta a pacientes nuevos en ambientes apropiados que proporcionen un marco adecuado para la intervención ${ }^{(28)}$. Otros hallazgos comunes entre los estudios previos y el nuestro es que los proveedores de la salud tienen gran carga de trabajo, que podría limitar su disposición a asumir responsabilidades adicionales ${ }^{(29)}$. Además, los proveedores no consideraron a las intervenciones breves, como parte de su papel, y prefieren remitir a los pacientes a otros especialistas, como los psiquiatras $(27,28)$. Pero esto último constituye un reto mayor, dada la escasez de establecimientos especializados en adicciones en el sistema público de servicios de salud en el Perú.

En conclusión, actualmente no existe un sistema que permita implementar el SBIRT de una manera que sea compatible con la rutina de proveedores de salud, que incluye a los internos de medicina, los médicos residentes, los médicos especialistas, las enfermeras y los trabajadores sociales. Con el fin de contar con políticas públicas exitosas que nos permitan diseñar las mejores estrategias de afronte, es necesario que se realicen investigaciones para conocer la prevalencia y los factores asociados al uso y abuso del alcohol y otras drogas en las PVVS en Perú. Asimismo, este estudio pone en evidencia la necesidad de desarrollar una red de referencia y contrarreferencia de tratamiento de drogas y alcohol en el sistema de salud peruano. Se requiere resolver la relación fragmentada entre la estrategia nacional de la salud mental y la estrategia nacional contra el VIH/ ITS con el fin de planificar las intervenciones conjuntas. Además, se recomienda futuras investigaciones con un enfoque del tipo "evaluación del desarrollo", donde se evalúen cambios sistemáticos y progresivos que puedan ayudar a implementar el modelo SBIRT para este tipo de pacientes, que involucren: 1) sensibilización y educación de los proveedores de atención a PVVS en la identificación y tratamiento del problema de abuso de sustancias; 2) Capacitación para aplicación del SBIRT realizada por profesionales de enfermería o psicólogos a las PVVS recientemente diagnosticados, y 3) trabajar con el Ministerio de Salud del gobierno peruano para implementar políticas que contemplen la problemática por abuso de alcohol y otras sustancias para mejorar las opciones del tratamiento.

Contribuciones de los autores: KF, FF JM y DMc han participado en la concepción y diseño del artículo. Además MC, LGF participaron en la recolección/obtención de datos. $\mathrm{KH}, \mathrm{JPT}, \mathrm{JB}, \mathrm{MC}, \mathrm{CB}, \mathrm{LS}$, LGF realizaron el análisis y la interpretación de los datos. Todos los autores participaron de la redacción y aprobación de la versión final.

Fuente de financiamiento: Instituto Nacional de Salud.

Conflictos de interés: los autores declaran no tener conflictos de interés. 


\section{REFERENCIAS BIBLIOGRÁFICAS}

1. Azar MM, Springer SA, Meyer JP, Altice FL. A systematic review of the impact of alcohol use disorders on HIV treatment outcomes, adherence to antiretroviral therapy and health care utilization. Drug Alcohol Depend. 2010;112(3):178-93. doi: 10.1016/j. drugalcdep.2010.06.014.

2. Altice FL, Bruce RD, Lucas GM, Lum PJ, Korthuis PT, Flanigan TP, et al. HIV treatment outcomes among HIV-infected, opioid-dependent patients receiving buprenorphine/ naloxone treatment within HIV clinical care settings: results from a multisite study. J Acquir Immune Defic Syndr. 2011;56 Suppl 1:S22-32. doi: 10.1097/ QAI.0b013e318209751e.

3. Chander G, Lau B, Moore RD. Hazardous alcohol use: a risk factor for non-adherence and lack of suppression in HIV infection. J Acquir Immune Defic Syndr. 2006;43(4):411-7.

4. Ángeles E, Factores asociados a fracaso al TARGA en pacientes con infección VIH en el Hospital Nacional Arzobispo Loayza-2009. Estudio Cualicuantitativo [Internet]. Lima: CVCC-VIH; 2011 [cited 2013 Oct7]. Disponible en: http:// cvcc-vih.com/estudios/study_detail/15

5. Lucas GM. Substance abuse, adherence with antiretroviral therapy, and clinical outcomes among HIV-infected individuals. Life Sci. 2011;88(2122):948-52. doi: $10.1016 / \mathrm{j}$. Ifs.2010.09.025.

6. Robinson-Papp J, Gelman BB, Grant I, Singer E, Gensler G, Morgello S, et al. Substance abuse increases the risk of neuropathy in an HIV-infected cohort. Muscle Nerve. 2012;45(4):471-6. doi: $10.1002 /$ mus. 23231.

7. Green JE, Saveanu RV, Bornstein RA. The effect of previous alcohol abuse on cognitive function in HIV infection. Am J Psychiatry. 2004;161(2):249-54.

8. Anand P, Springer SA, Copenhaver MM, Altice FL. Neurocognitive impairment and HIV risk factors: a reciprocal relationship. AIDS Behav. 2010;14(6):1213-26. doi: 10.1007/ s10461-010-9684-1.

9. Sullivan LE, Saitz R, Cheng DM, Libman $\mathrm{H}$, Nunes D, Samet JH. The impact of alcohol use on depressive symptoms in human immunodeficiency virus-infected patients. Addiction. 2008;103(9):14617. doi: $10.1111 /$ j.13600443.2008.02245.x

10. Cheng DM, Libman $H$, Bridden C, Saitz R, Samet JH. Alcohol consumption and lipodystrophy in HIV-infected adults with alcohol problems. Alcohol.
2009;43(1):65-71. doi: 10.1016/j. alcohol.2008.09.004.

11. Cheng DM, Nunes D, Libman $\mathrm{H}$, Vidaver J, Alperen JK, Saitz R, et al. Impact of hepatitis C on HIV progression in adults with alcohol problems. Alcohol Clin Exp Res. 2007;31(5):829-36.

12. Shuper PA, Joharchi N, Irving H, Rehm $\mathrm{J}$. Alcohol as a correlate of unprotected sexual behavior among people living with HIV/AIDS: review and meta-analysis. AIDS Behav. 2009;13(6):1021-36. doi: 10.1007/s10461-009-9589-z.

13. Wand H, Guy R, Law M, Wilson DP, Maher L. High Rates of Late HIV Diagnosis Among People Who Inject Drugs Compared to Men Who Have Sex with Men and Heterosexual Men and Women in Australia. AIDS Behav. 2013;17(1):235-41. doi: 10.1007/ s10461-011-0117-6.

14. DeLorenze GN, Weisner C, Tsai A-L, Satre DD, Quesenberry CP Jr. Excess mortality among HIV-infected patients diagnosed with substance use dependence or abuse receiving care in a fully integrated medical care program. Alcohol Clin Exp Res. 2011; 35(2):203-10. doi: 10.1111/j.1530-0277.2010.01335.x.

15. World Health Organization. Clinical guidance across the continuum of care: managing common coinfections and comorbidities [Internet]. Geneva: WHO; 2013 [cited 2013 Dec 26]. Disponible en: http://www.who.int/hiv/ pub/guidelines/arv2013/coinfection/ en/index.htm

16. Miller WR, Rollnick S. Ten things that motivational interviewing is not. Behav Cogn Psychother. 2009;37(2):129-40. doi: $10.1017 / S 1352465809005128$.

17. Rollnick S, Miller WR, Butler CC, Aloia MS. Motivational Interviewing in Health Care: Helping Patients Change Behavior. COPD J Chronic Obstr Pulm Dis. 2008;5(3):203.

18. Mbuagbaw L, Ye C, Thabane L. Motivational interviewing for improving outcomes in youth living with HIV. Cochrane Database Syst Rev. 2012;9:CD009748. doi: 10.1002/14651858.CD009748.pub2.

19. Smedslund G, Berg RC, Hammerstrøm KT, Steiro A, Leiknes KA, Dahl HM, et al. Motivational interviewing for substance abuse. Cochrane Database Syst Rev. 2011;(5):CD008063. doi: 10.1002/14651858.CD008063.pub2.

20. Rubak S, Sandbaek A, Lauritzen T, Christensen B.Motivational interviewing: a systematic review and meta-analysis. Br J Gen Pract. 2005;55(513):305-12.
21. Murphy DA, Chen $X$, Naar-King $S$, Parsons JT, Adolescent Trials Network. Alcohol and Marijuana Use Outcomes in the Healthy Choices Motivational Interviewing Intervention for HIVPositive Youth. AIDS Patient Care STDs. 2012;26(2):95-100. doi: 10.1089/ apc.2011.0157.

22. Ino A, Cho T. [Significance of SBIRT and countermeasures for its dissemination]. Nihon Arukōru Yakubutsu Igakkai Zasshi. 2013;48(2):105-17.

23. McCormick KA, Cochran NE, Back AL, Merrill JO, Williams EC, Bradley KA. How primary care providers talk to patients about alcohol: a qualitative study. J Gen Intern Med. 2006;21(9):966-72.

24. Johnson M, Jackson R, Guillaume L, Meier P, Goyder E. Barriers and facilitators to implementing screening and brief intervention for alcohol misuse: a systematic review of qualitative evidence. J Public Health (Oxf). 2011; 33(3):41221. doi: 10.1093/pubmed/fdq095.

25. Williams EC, Johnson ML, Lapham GT, Caldeiro RM, Chew L, Fletcher GS, et al. Strategies to implement alcohol screening and brief intervention in primary care settings: a structured literature review. Psychol Addict Behav. 2011;25(2):20614. doi: 10.1037/a0022102.

26. Bowen DJ, Kreuter M, Spring B, CoftaWoerpel L, Linnan L, Weiner D, et al. How we design feasibility studies. Am J Prev Med. 2009;36(5):452-7. doi: 10.1016/j.amepre.2009.02.002.

27. Johnson M, Jackson R, Guillaume L, Meier P, Goyder E. Barriers and facilitators to implementing screening and brief intervention for alcohol misuse: a systematic review of qualitative evidence. J Public Health. 2011;33(3):412-21. doi: 10.1093/pubmed/fdq095.

28. Heather N, Dallolio E, Hutchings D, Kaner E, White M. Implementing routine screening and brief alcohol intervention in primary health care: A Delphi survey of expert opinion. J Subst Use. 2004;9(2):68-85.

29. Johansson K, Akerlind I, Bendtsen P. Under what circumstances are nurses willing to engage in brief alcohol interventions? A qualitative study from primary care in Sweden. Addict Behav. 2005;30(5):1049-53.

\section{Correspondencia: Kim Hoffman}

Dirección: Calle Diego Ferre 218, Miraflores, Lima Perú.

Teléfono: 975542159

Correo electrónico:kim@incaas.org 